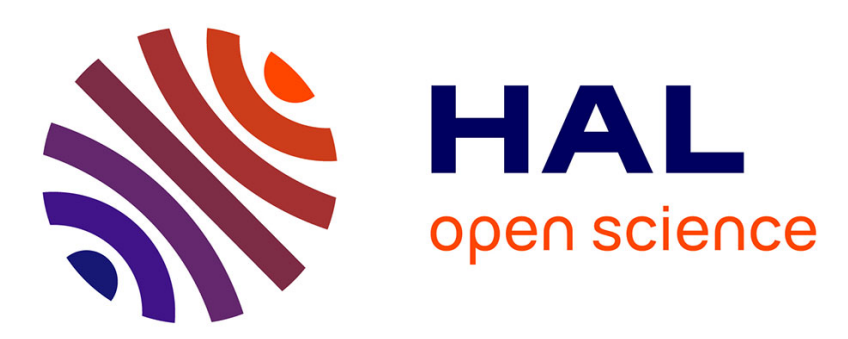

\title{
Multi-scale finite-strain plasticity model for stable metallic honeycombs incorporating microstructural evolution
}

\author{
Dirk Mohr
}

\section{- To cite this version:}

Dirk Mohr. Multi-scale finite-strain plasticity model for stable metallic honeycombs incorporating microstructural evolution. International Journal of Plasticity, 2006, 22, pp.1899-1923. 10.1016/j.ijplas.2006.01.004 . hal-00111456

\section{HAL Id: hal-00111456 https://hal.science/hal-00111456}

Submitted on 1 Mar 2019

HAL is a multi-disciplinary open access archive for the deposit and dissemination of scientific research documents, whether they are published or not. The documents may come from teaching and research institutions in France or abroad, or from public or private research centers.
L'archive ouverte pluridisciplinaire HAL, est destinée au dépôt et à la diffusion de documents scientifiques de niveau recherche, publiés ou non, émanant des établissements d'enseignement et de recherche français ou étrangers, des laboratoires publics ou privés. 


\title{
Multi-scale finite-strain plasticity model for stable metallic honeycombs incorporating microstructural evolution
}

\author{
Dirk Mohr \\ Impact and Crashworthiness Laboratory, Massachusetts Institute of Technology, Cambridge, MA, USA \\ Department of Mechanics, CNRS UMR 7649, Ecole Polytechnique, Palaiseau, France
}

This paper deals with the development of a mechanism-based two-scale constitutive model for thick-walled metallic honeycombs in sandwich applications. The mechanical response of metallic honeycomb sandwich sheets subject to large in-plane normal loading and out-of-plane shear loading is investigated using a detailed finite element model of the honeycomb microstructure. Based on the simulation results, a simple micro-mechanical system is proposed and used to develop the macroscopic constitutive model. The finite-strain constitutive model accounts for microstructural evolution due to geometrical changes and strain hardening at the microscale. The macroscopic model has been validated for various loading conditions. Furthermore, the evolution of the macroscopic yield surface for pure out-of-plane shear is discussed in detail.

Keywords: Honeycombs; Sandwich structures; Constitutive modeling; Large deformations; Plasticity; Finite strains

\section{Introduction}

Metallic honeycombs exhibit highly orthotropic periodic microstructures composed of tubular cells. This type of material finds widespread use in lightweight vehicle structures, notably as core material in sandwich constructions. The mechanical behavior of metallic honeycombs depends strongly on their so-called microstructural configuration: the cell shape, cell size, cell wall thickness and the cell wall base material properties. Thin-walled 
honeycombs of relative densities below 5\% are mostly used in aerospace engineering. These materials combine low weight with substantial out-of-plane shear stiffness and strength. Ultralight honeycombs are also employed in energy absorption devices; upon compressive loading along their tubular direction, thin-walled honeycomb microstructures respond by the folding of the cell walls, a process which is characterized by an almost constant force level at the macroscale (e.g. Gibson and Ashby, 1997). The response of thin-walled honeycombs to in-plane loading is characterized by the formation of bands of collapsing cells within the twodimensional microstructure (e.g., Zhang and Ashby, 1992; Papka and Kyriakides, 1999). Theoretical models have been developed to predict the occurrence of buckling-type instabilities in low density honeycombs subject to general in-plane loading (e.g., Triantafyllidis and Schraad, 1998; Okumura et al., 2004). Mohr and Doyoyo (2004) performed multi-axial experiments to come up with a physically-motivated model to describe the macroscopic behavior of a low density aluminum honeycomb in crashworthiness applications. Quasi-static experiments on low-density honeycombs including non-proportional loading paths have been carried out by Hong et al. (2006, in press). Based on finite element simulations, Xue et al. (submitted) proposed a phenomenological constitutive model for thin-walled honeycombs incorporating strain-rate dependency (Zhao and Gary, 1998). It shall be noted that the yield surfaces for constructed cellular solids such as honeycombs and truss lattice materials may exhibit corners in the six-dimensional stress space, whereas the random nature and different microstructural deformation mechanisms results in more smooth yield surfaces for foams (e.g., Doyoyo and Wierzbicki, 2003; Wang and Pan, in press; Zhang and Lee, 2003).

Thick-walled honeycombs of relative densities above $10 \%$ are of growing interest in automotive applications. However, unlike their thin-walled counterparts, the microstructure of thick-walled honeycombs remains 'stable' when subject to mechanical loading. Instead of forming folds, the cell walls of thick-walled honeycombs are being deformed in a fairly uniform manner while they remain essentially flat. It is emphasized that this fundamental difference between thin-walled and thick-walled honeycombs is of foremost importance in modeling the mechanical behavior of this type of materials. Based on the assumption that plastic yield at the microscale defines the onset of macroscopic failure, Wang and McDowell (2005) derived the three-dimensional failure surfaces for periodic metal honeycombs at intermediate density.

In this work, a micromechanics-based constitutive model for thick-walled metallic honeycombs is developed. Specific characteristics of the honeycomb microstructure such as the cell wall thickness, shape and orientation are taken into account which provides the model with some predictive capabilities. Throughout the development, special emphasis is placed on the coupling between large in-plane deformation and the evolution of the out-of-plane shear strength. This paper is organized as follows: a detailed finite element model of the characteristic 'unit cell' of the honeycomb sandwich material is presented in Section 2 and subsequently used to study the macroscopic response of a thick-walled honeycomb under various loading conditions. Based on the microstructural simulation results, a simple mechanical system is proposed in Section 3 to model the governing deformation mechanisms at the microscale. With this simple micromechanical system in mind, a macroscopic finitestrain constitutive model is formulated for thick-walled honeycomb core materials and implemented into a commercial finite element code. In Section 4, the macroscopic constitutive model is calibrated and validated by comparison with the response of the detailed finite element model. Furthermore, the evolution of the macroscopic yield surface of the honeycomb material is discussed in Section 5. 


\section{Material and virtual experiments}

In view of practical applications, we consider an all-metal honeycomb sandwich sheet which has been shown to be formable (see Mohr and Straza, 2005). This material comprises a $12.5 \%$ relative density honeycomb core and two $0.2 \mathrm{~mm}$ thick face sheets. Fig. 1 shows a schematic of the periodic microstructure. The cell walls are $l=0.92 \mathrm{~mm}$ wide and $t=0.075 \mathrm{~mm}$ thick, which corresponds to a width-to-thickness ratio of $l / t \cong 12$. The cell wall opening angle for this perfectly hexagonal microstructure is $\theta=30^{\circ}$. Due to the manufacturing process, the cell walls aligned with the $L$-direction are of double-thickness. Throughout our discussion, the in-plane direction perpendicular to the $L$-axis is referred to as $W$-direction.

The plastic properties of the face sheets (stainless steel 347) and the cell wall base material (hastelloy X) have been determined from uniaxial tensile tests (Fig. 2). Both materials exhibit pronounced strain hardening which contributes to the microstructural stability of the sandwich material (e.g., prevention of local plastic buckling). Furthermore, an elastic modulus of $E=205 \mathrm{GPa}$ and a Poisson's ratio of $v=0.33$ are assumed for both parent materials. The overall behavior of the sandwich material is determined from 'virtual experiments', i.e., finite element simulations of a characteristic unit cell of the sandwich structure. The shaded rectangle in Fig. 1 highlights the basic ' $Y$ '-unit which makes up the entire hexagonal microstructure.

\subsection{Detailed shell element model}

Two neighboring Y-units are chosen as mechanical unit cell in investigating the mechanical behavior of the sandwich material (Fig. 3a). This particular unit cell is chosen to facilitate the prescription of periodic displacement boundary conditions. The unit cell widths along the $W$ - and $L$-direction are $\Delta W=1.6 \mathrm{~mm}$ and $\Delta L=2.772 \mathrm{~mm}$, respectively. The sandwich core height, that is the unit cell length along the $T$-direction, is $\Delta T=2.2 \mathrm{~mm}$. The entire unit cell geometry is discretized by 4-node reduced-integration shell elements of three different thicknesses (Fig. 3a): $0.2 \mathrm{~mm}$ for the top and bottom face sheets (light grey), $0.150 \mathrm{~mm}$ for the cell walls aligned with the $L$-direction (white), and $0.075 \mathrm{~mm}$ for the single-thickness cell walls (dark grey).

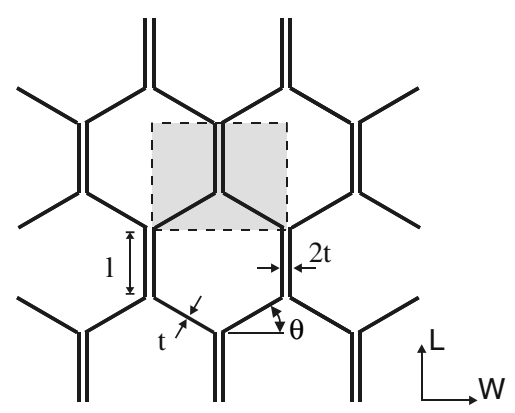

Fig. 1. Schematic of the (perfectly) hexagonal honeycomb microstructure. The dashed rectangle highlights the basic Y-unit cell. The double-thickness cell walls are initially aligned with the $L$-direction. 


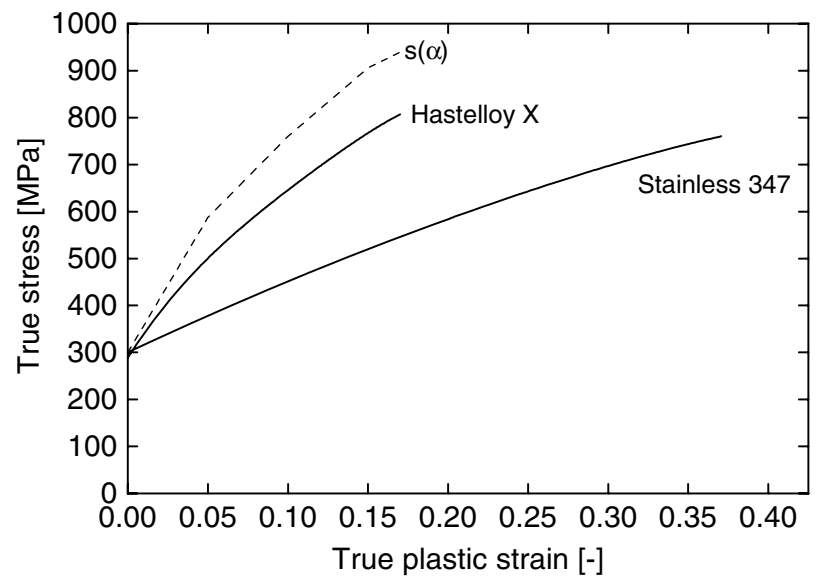

Fig. 2. True stress-strain curves of the cell wall material (Hastelloy X) and the face sheets (stainless steel 347). Both curves have been determined from uniaxial tensile tests on a prototype sandwich sheet material (Aerovision, San Diego, CA). The dashed line shows the cell wall base material stress-strain curve obtained from calibration of the constitutive model.
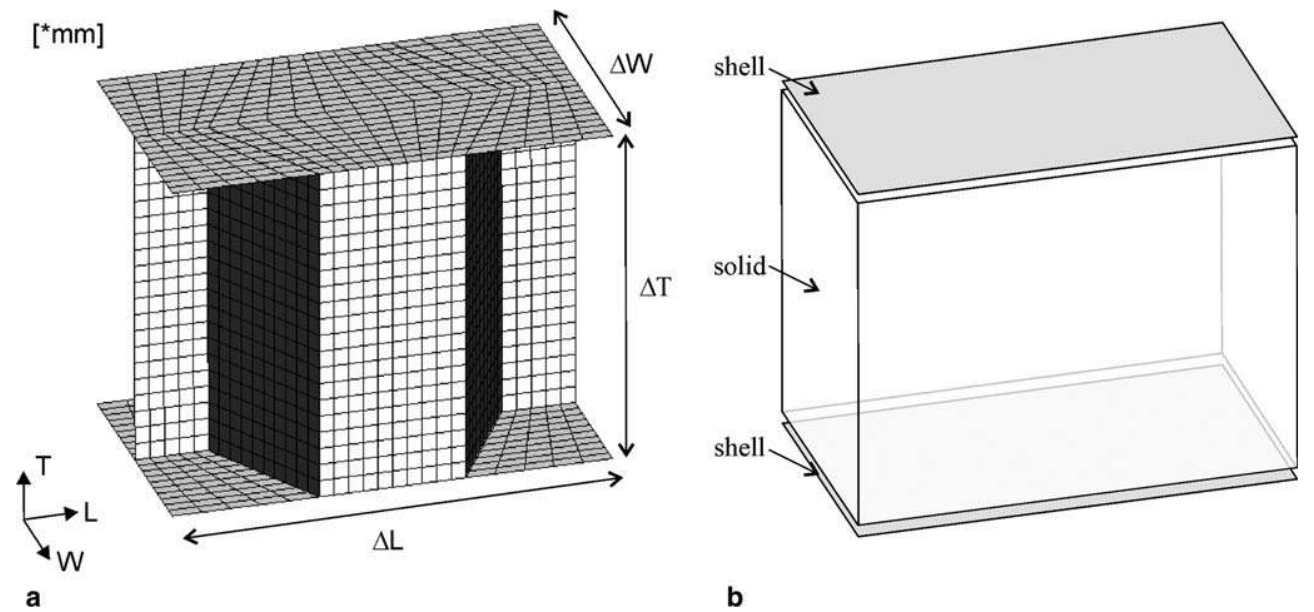

Fig. 3. (a) Detailed finite element model of a period unit of the honeycomb sandwich, three different gray shades indicate the regions of homogeneous shell element thickness; (b) schematic of the continuum finite element model comprising solid elements for the honeycomb core and shell elements for the face sheets.

Periodic boundary conditions are defined for the nodes at the boundaries of the unit cell. Additionally, the average displacements are prescribed on these boundaries. The notation $\left\langle u_{i}\right\rangle^{x_{j}=a}$ is introduced to denote the average of the displacement component $u_{i}$ at the boundary surface defined by all nodes for which the initial coordinate $x_{j}=a$. Using this notation, we define the macroscopic in-plane (engineering) normal strains 


$$
\begin{aligned}
E_{L L} & =\frac{\left\langle u_{L}\right\rangle^{x_{L}=\Delta L}-\left\langle u_{L}\right\rangle^{x_{L}=0}}{\Delta L}-1 \\
E_{W W} & =\frac{\left\langle u_{W}\right\rangle^{x_{W}=\Delta W}-\left\langle u_{W}\right\rangle^{x_{W}=0}}{\Delta W}-1
\end{aligned}
$$

and the macroscopic out-of-plane (engineering) shear strains

$$
\begin{aligned}
E_{L T} & =\frac{\left\langle u_{L}\right\rangle^{x_{T}=\Delta T}-\left\langle u_{L}\right\rangle^{x_{T}=0}}{\Delta T} \\
E_{W T} & =\frac{\left\langle u_{W}\right\rangle^{x_{T}=\Delta T}-\left\langle u_{W}\right\rangle^{x_{T}=0}}{\Delta T}
\end{aligned}
$$

\subsection{Virtual experiments}

Finite element simulations are performed to investigate the honeycomb sandwich sheet behavior under selected loading conditions which are important in sandwich forming operations. In particular, the interaction between in-plane deformation and out-of-plane shear is studied in detail.

\subsubsection{In-plane compression and tension}

When subject to uniaxial in-plane loading, incompatibilities between the unconstrained deformation responses of the honeycomb core material and the face sheets may lead to additional stresses within the sandwich material. For flat face sheets made of a Levyvon Mises material, simple relationships between the in-plane normal strain components $E_{W W}$ and $E_{L L}$ may be derived from the condition of plastic incompressibility. In a first approximation, we neglect elastic deformations and enforce incompressibility by setting the trace of the true strain tensor to zero. Thus, as the engineering strain $E_{W W}$ is prescribed (uniaxial loading along the $W$-direction), the reaction strain along the $L$-direction due to plastic incompressibility is

$$
E_{L L}=\frac{1}{\sqrt{1+E_{W W}}}-1
$$

Similarly, we have the reaction strain $E_{W W}$ upon uniaxial (monotonic) loading along the $L$-direction:

$$
E_{W W}=\frac{1}{\sqrt{1+E_{L L}}}-1
$$

Analogous expressions may be derived for the honeycomb core material. For this, we assume that the honeycomb material deforms preferentially by the formation of plastic hinges (bending-dominated) rather than uniform stretching/compressing of its cell walls. Using the simple hinge model shown in Fig. 4, we obtain the following relationship between the in-plane strain components $E_{L L}$ and $E_{W W}$ :

$$
E_{L L}=\frac{1+\sqrt{1-\cos ^{2} \theta\left(1+E_{W W}\right)^{2}}}{1+\sin \theta}-1
$$



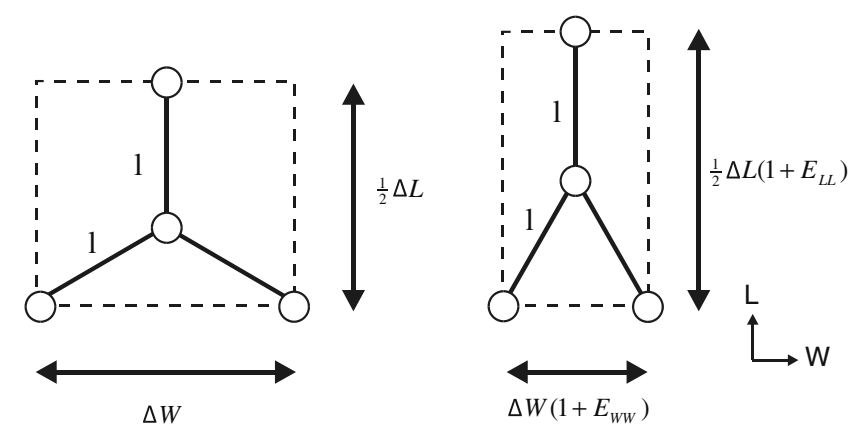

Fig. 4. Low-resistance in-plane deformation mode of the honeycomb core. The microstructure deforms by forming plastic hinges at the cell wall intersections whereas the cell wall length remains constant throughout deformation.

Recall that $\theta$ denotes the initial cell wall opening angle. We note that Eq. (7) may be applied to both cases of prescribed deformation along the $W$-and $L$-axis. Fig. 5 shows a comparison of the preferential in-plane deformation modes of the face sheets and the honeycomb material. Both plots are used in the following to explain some of the results obtained from detailed finite element analysis.

2.2.1.1. Uniaxial loading along the $W$-direction. Simulations are carried out for both uniaxial compression and tension along the $W$-direction. Fig. 6a shows a plot of the section force $N_{W}$ as a function of the applied work-conjugate strain $E_{W W}$. We divided $N_{W}$ by the initial cross-sectional area $(\Delta T)(\Delta L)$, i.e., $N_{W} /(\Delta T)(\Delta L)$ may be interpreted as the mean normal stress in the $W$-direction. In addition to the results from the detailed finite element model of the sandwich sheet (solid line), we plotted the response of a fictitious sandwich material comprised of face sheets only, that is a sandwich sheet where the core has zero resistance to deformation (dashed line). Fig. 6 also shows the curves for a continuum model which will be discussed in detail in Section 4. The comparison of these two results indicates that both the solid and dashed curves are remarkably close in the compressive range $\left(E_{W W}<0\right)$, while the section force for the honeycomb sandwich sheet is substantially higher as tension is applied $\left(E_{W W}>0\right)$. In other words, the core material's contribution to the section force may be neglected under compression, whereas its contribution must be taken into account when tension is applied. This observation may be explained by the lack of compatibility between the core and face sheet deformation. As illustrated in Fig. 5a, the unconstrained deformation mode of the face sheets and the bending-dominated low-resistance deformation mode of the honeycomb core are fairly compatible in the compressive range. However, in the tensile regime $\left(E_{W W}>0\right)$, the lateral contraction $\left(E_{L L}<0\right)$ of the core material is significantly larger than the plastic Poisson effect for the face sheet material. Thus, additional work is required to achieve mechanical compatibility between the core and face sheets, which explains the increased deformation resistance of the sandwich section when subject to tensile loading along the $W$-direction. More specifically, we note that the cell walls are being compressed and locally bent to match the deformation field of the face sheet. For the same reason, tensile stresses act on the face sheets in the $L$-direction (in addition to tension along the $W$-direction). 

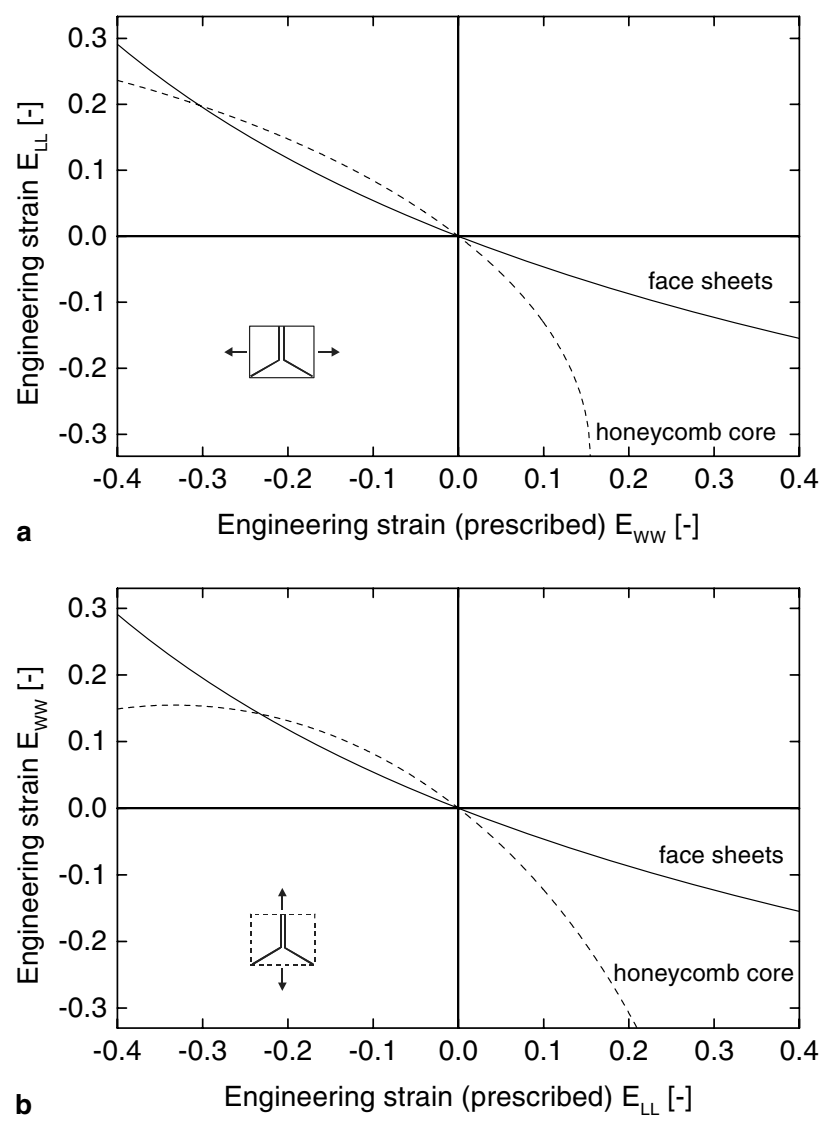

Fig. 5. Incompatibility of in-plane deformation. The solid curves show the Poisson deformation of the face sheets under uniaxial loading (prescribed strain), whereas the dashed lines depict the low-resistance-mode in-plane strain interaction curve for the idealized honeycomb core structure.

2.2.1.2. Uniaxial loading along the L-direction. Similar results have been obtained from the detailed finite element analysis of the unit cell subject to uniaxial compression or tension along the $L$-direction. As for loading along the $W$-direction, the comparison of the honeycomb sandwich response with the results for face sheets only (Fig. 6b) reveals only little difference for compression whereas the tensile force $N_{L} /(\Delta T)(\Delta W)$ is remarkably higher. However, as far as the microstructural deformation fields are concerned, it is worth noting that the face sheet dimpling occurred under large compressive loads. Observe from Fig. 5b that the incompatibility between the core and face sheet deformation fields is expected to laterally compress the face sheets for large strains along the $L$-direction $\left(E_{L L}<-0.25\right)$. This effect of biaxial face sheet compression may also be expected upon compressive loading along the $W$-direction, but it occurs later and is less pronounced (see Fig. 5a). In addition to the development of biaxial compression, geometrical changes at the microstructural level increase the likelihood of buckling for large strains along the $L$-direction. Fig. 7 shows the top view of the sandwich sheets after various uniaxial loading scenarios. The solid lines highlight the free edges of the face sheets as well as the intersection 

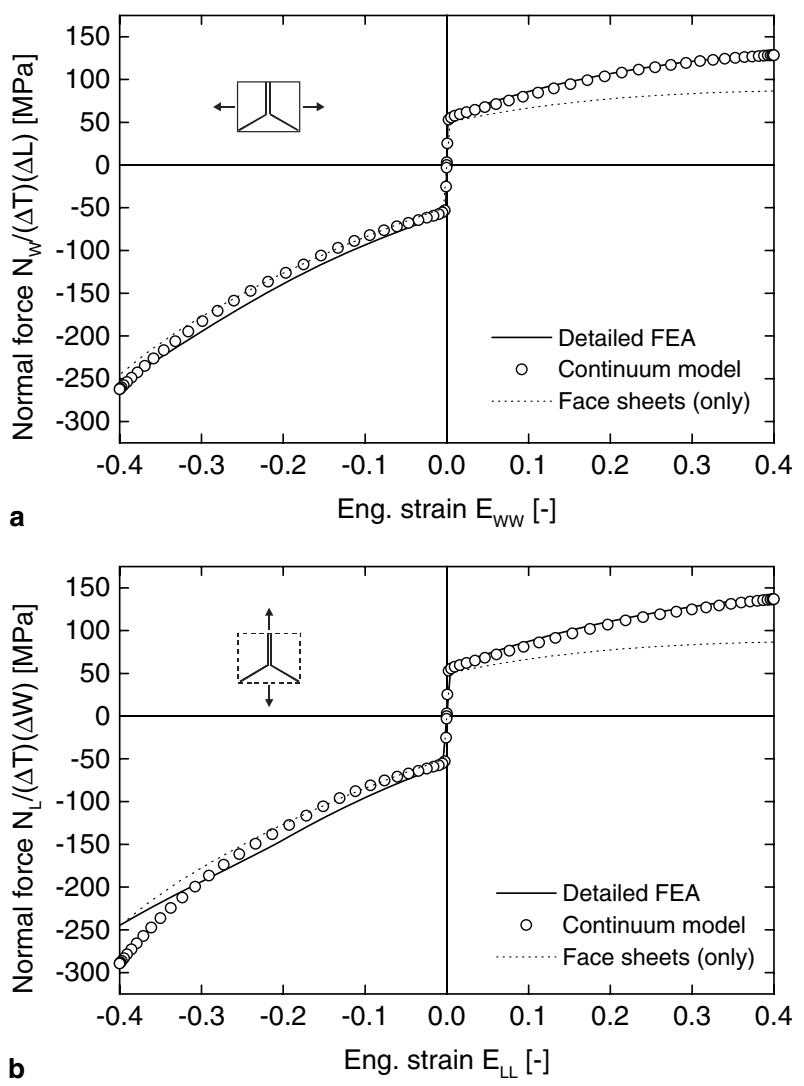

Fig. 6. Cross-section force normalized by the cross-sectional area as a function of the prescribed work-conjugate macroscopic in-plane strains: (a) uniaxial loading along the $W$-direction; (b) uniaxial loading along the $L$ direction.

lines between the top face sheet and the honeycomb cell walls. The comparison of Fig. 7a (compression along $L$-axis) and Fig. 7c (compression along the $W$-axis) reveals that uniaxial loading along the $L$-axis transforms the hexagonally-shaped cell into a rectangle. On the other hand, uniaxial loading along the $W$-direction produces an elongated hexagonal cell. It is speculated that the rectangular shape facilitates the dimpling of the face sheets. Regarding the orthotropy of the sandwich material, we note that the mechanical response to uniaxial in-plane loading along the $W$ - and $L$-axis is fairly similar.

\subsubsection{Out-of-plane shear loading}

Out-of-plane shear loading corresponds to the relative motion of the sandwich face sheets in the $L$ - and $W$-direction. In order to investigate the effect of in-plane loading history, we subject both initially undeformed and previously-deformed honeycomb sandwich configurations to shear loading.

2.2.2.1. Shear loading in the $T$ - $L$-plane. A moderate macroscopic shear strain of $E_{L T}=0.1$ is applied to the initially undeformed sandwich material. Furthermore, shear loading is 

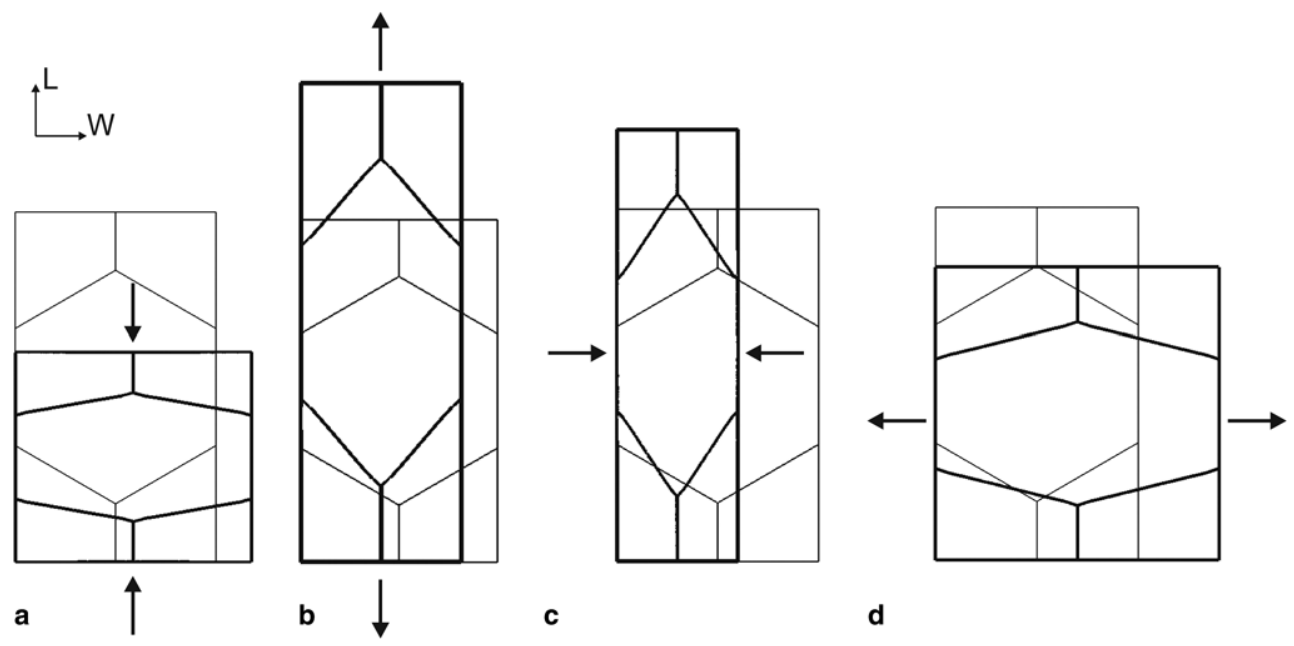

Fig. 7. Microstructural evolution under uniaxial in-plane loading: $E_{W W}=-0.4$; (d) $E_{W W}=0.4$.

applied to configurations which have been previously subject to large uniaxial compressive and tensile loading along the $W$-direction $\left(E_{W W}=-0.4\right.$ and $\left.E_{W W}=0.4\right)$. Fig. 8 shows the final configurations after shear loading along the $L$-direction. The solid curves in Fig. 10a show the corresponding shear stress versus shear strain curves. In Fig. 10a, the macroscopic (engineering) shear stress is defined as $S_{L T}=F_{L} /(\Delta W)(\Delta L)$, where $F_{L}$ denotes the total shear force acting on the top face sheet. The curve for $E_{W W}=0$ (i.e., initially undeformed unit cell) exhibits a well-defined yield point at about $14 \mathrm{MPa}$, while the predeformed microstructures $\left(E_{W W}= \pm 0.4\right)$ responded in a non-linear manner even for fairly small macroscopic shear strains. Clearly, the deformation history has a strong influence on the shear response of the honeycomb core, both quantitatively and qualitatively. The initially undeformed material $\left(E_{W W}=0\right)$ exhibits the lowest shear force level. Observe that the force level for the pre-tensioned $\left(E_{W W}=0.4\right)$ and the pre-compressed $\left(E_{W W}=-0.4\right)$ sandwich material are respectively up to 5 and $20 \mathrm{MPa}$ higher. These differences are due to the local strain hardening of the cell wall material as well as geometrical changes which have been introduced by the preceding in-plane deformation.

2.2.2.2. Shear loading in the $T-W$-plane. Fig. 9 shows the honeycomb microstructures after out-of-plane shear loading along the $W$-direction. Here, a macroscopic shear strain of $E_{W T}=0.1$ is applied to the initially undeformed microstructure $\left(E_{L L}=0\right)$ and to two pre-deformed microstructures which have been subject to in-plane compression and tension along the $L$-direction $\left(E_{L L}=-0.4\right.$ and $\left.E_{L L}=0.4\right)$. The corresponding shear stress-strain curves are depicted by the solid lines in Fig. 10b. The initially undeformed microstructure yielded at a macroscopic shear stress of about $S_{W T}=9 \mathrm{MPa}$ which is significantly lower than the yield stress for shear loading in the $L$-direction. Recall that the sandwich material exhibited only little orthotropy in its mechanical in-plane behavior, whereas the orthotropy of the honeycomb core becomes more dominant when subject to out-of-plane shear. 


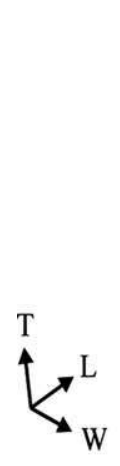

a

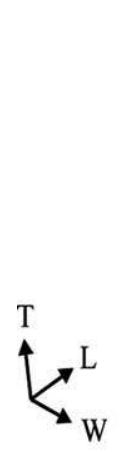

b

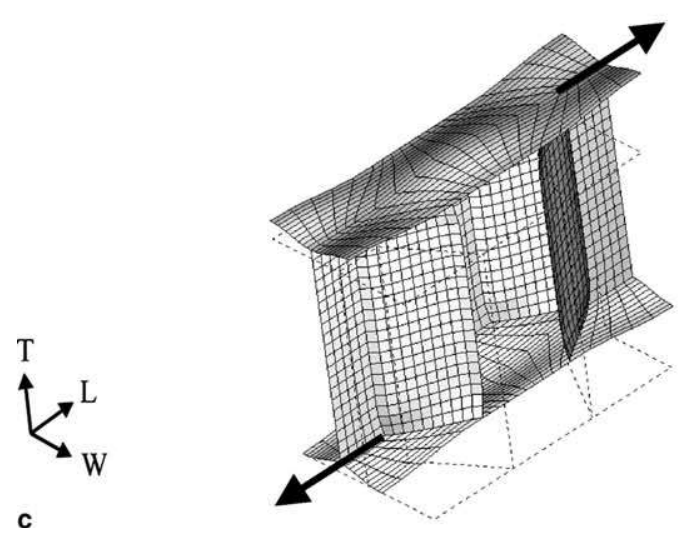

Fig. 8. Out-of-plane shear loading along the $L$-direction: (a) without deformation history $\left(E_{W W}=0\right)$; (b) after uniaxial tension along the $W$-direction $\left(E_{W W}=0.4\right)$; (c) after uniaxial compression along the $W$-direction $\left(E_{W W}=-0.4\right)$.

In a first approximation, it may be assumed that the shear loads in the $W$-direction are primarily carried by the single-thickness cell walls which are oriented at some angle with respect to the loading direction. When the sandwich structure has been pre-deformed 

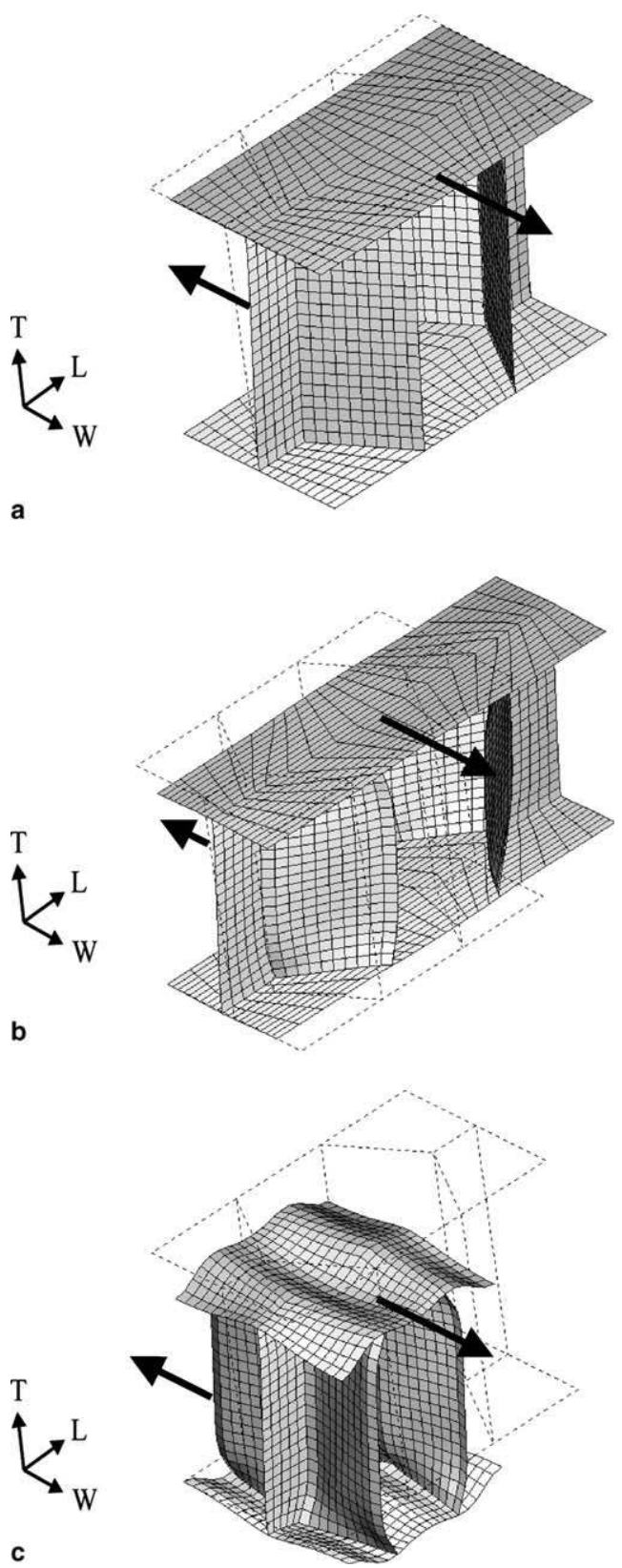

Fig. 9. Out-of-plane shear loading along the $W$-direction: (a) without deformation history $\left(E_{L L}=0\right.$ ); (b) after uniaxial tension along the $L$-direction $\left(E_{L L}=0.4\right)$; (c) after uniaxial compression along the $L$-direction $\left(E_{L L}=-0.4\right)$.

under in-plane compression (Fig. 7a), these cell walls are almost aligned with the $W$-direction. This geometrical change is expected to contribute to the observed increase in the macroscopic out-of-plane shear resistance (Fig. 10b). In the case of the pre-tensioned 

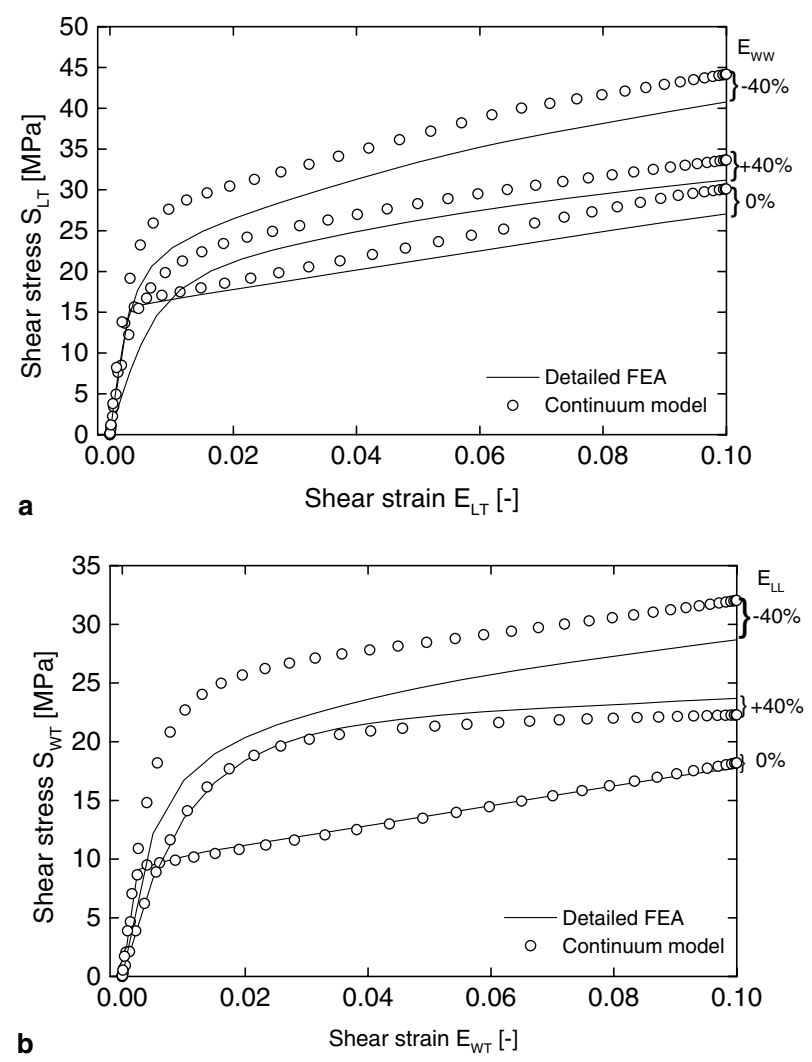

Fig. 10. Macroscopic stress-strain curves for out-of-plane shear loading. For each loading combination, the graphs show the simulation results for the detailed unit cell model (solid lines) and the continuum model (open circles). (a) Out-of-plane shear along the $L$-direction after uniaxial in-plane loading along the $W$-direction $\left(E_{W W}=0.0,+0.4,-0.4\right)$; (b) out-of-plane shear along the $W$-direction after uniaxial in-plane loading along the $L$-direction $\left(E_{L L}=0.0,+0.4,-0.4\right)$.

microstructure $\left(E_{L L}=0.4\right)$, the shear force level is increased even further which may be attributed to substantial cell wall material hardening. Recall that the face sheet-core incompatibility causes cell wall stretching during in-plane tensile loading.

\section{Continuum model}

In this section, an equivalent continuum model is developed to simulate the macroscopic behavior of the honeycomb sandwich material. In the continuum model, the sandwich core is represented by solid elements along with an appropriate macroscopic constitutive model of the core material. The sandwich facings on the other hand are still modeled using shell elements (Fig. 3b). No attempt will be made at the continuum level to describe local face sheet deformation modes such as wrinkling or dimpling.

A micromechanics-based approach is used to obtain the macroscopic constitutive equations. It is fairly difficult to come up with a computationally-efficient constitutive model for the core material when the honeycomb is considered as a shell structure at the 
microscale (such as the detailed finite element model). Here, the idea is to consider each cell wall as membrane which significantly reduces the complexity of the modeling approach. From a physical point of view, this strong simplification is motivated by the following observations:

(1) The cell walls of a thick-walled honeycomb remain more or less flat when subject to large macroscopic deformations (see Figs. 8 and 9).

(2) The plastic dissipation due to the relative rotation of the cell walls appears to be negligible as compared to the work required to uniformly stretch or compress the flat cell walls. This assumption is supported by the observation that the core resistance may be neglected when its bending-dominated mode is compatible with the face sheet kinematics (e.g., in-plane compression), whereas considerable work is required when compatibility is achieved through cell wall stretching (e.g., macroscopic inplane tension, see Fig. 6).

The model development involves two scales: at the macroscopic scale, the honeycomb material is considered as a homogeneous medium, whereas at the microscale, the discrete cell (membrane) structure is taken into account. Given a macroscopic deformation gradient, we will solve the so-called 'local problem' and then translate the results from the micro- to the macroscale.

Throughout this presentation, we use the notation of modern continuum mechanics (e.g., Gurtin, 1981). Specifically, a $\mathbf{b}$ denotes the scalar (inner) product of two vectors a and $\mathbf{b}$; the same symbol is used to denote the inner product of two second-order tensors; $\mathbf{a} \otimes \mathbf{b}$ denotes the dyadic (tensor) vector product, that yields a linear transformation (second-order tensor) defined as $(\mathbf{a} \otimes \mathbf{b}) \mathbf{c}=(\mathbf{c} \cdot \mathbf{b}) \mathbf{a}$, where $\mathbf{c}$ is a vector of the same dimension as $\mathbf{a}$ and $\mathbf{b}$. Vectors with components $\left[\begin{array}{lll}1 & 0 & 0\end{array}\right]^{\mathrm{T}},\left[\begin{array}{lll}0 & 1 & 0\end{array}\right]^{\mathrm{T}}$, and $\left[\begin{array}{lll}0 & 0 & 1\end{array}\right]^{\mathrm{T}}$ are denoted as $\mathbf{e}_{1}, \mathbf{e}_{2}$, and $\mathbf{e}_{3}$, respectively. (Note. The superscript ' $\mathrm{T}$ ' denotes the transpose of a vector or matrix, whereas the subscript ' $\mathrm{T}$ ' indicates the coordinate axis $T$ ).

\subsection{Kinematics}

When considering each honeycomb cell wall as flat membrane, the basic Y-unit cell may be used to estimate the macroscopic material behavior. The schematic in Fig. 11 illustrates the deformation of a Y-element subject to a macroscopic deformation field. At the macroscale, we introduce the deformation gradient $\mathbf{F}$ to describe the one-to-one mapping of an infinitesimal vector from the initial configuration, $\mathbf{d X}$, to the deformed configuration, $\mathbf{d x}$,

$$
\mathbf{d x}=\mathbf{F}(\mathbf{d X})
$$

At the microscale, the initial configuration of the honeycomb microstructure is uniquely defined by four unit vectors: the vector $\mathbf{e}_{T}^{0}$, which is aligned with the $T$-direction, and the three vectors $\mathbf{n}_{r}^{0}, r=1,2,3$, which define the in-plane orientations of the cell walls (Fig. 11). Observe that $\mathbf{n}_{3}^{0}$ is associated with the double-thickness cell wall. To describe the evolution of the microstructure, it is convenient to introduce the affine configuration defined by the four (non-unit) vectors $\hat{\mathbf{e}}_{T}, \hat{\mathbf{n}}_{1}, \hat{\mathbf{n}}_{2}$, and $\hat{\mathbf{n}}_{3}$, where

$$
\hat{\mathbf{e}}_{T}=\mathbf{F e}_{T}^{0}, \quad \hat{\mathbf{n}}_{r}=\mathbf{F n}_{r}^{0} \quad \text { for } r=1,2,3
$$



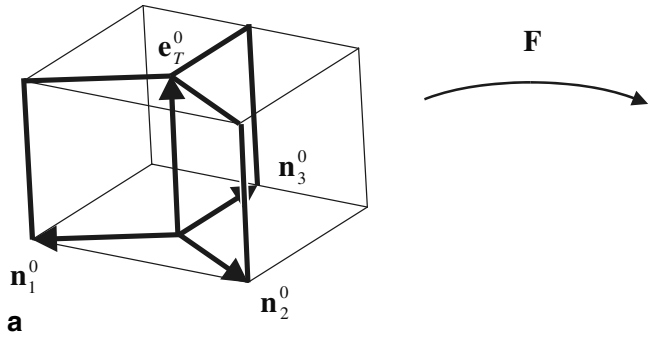

a

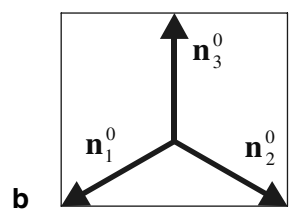

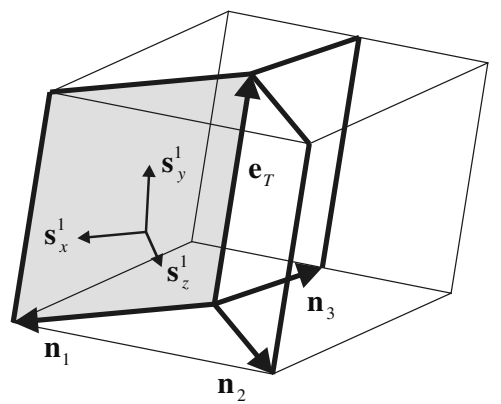

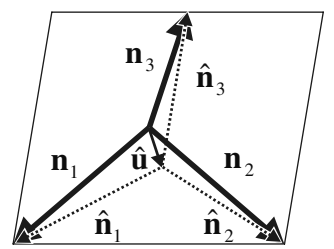

Fig. 11. Kinematics of the three-membrane unit cell: (a) 3D view; (b) in-plane deformation. The affine configuration is only shown in ' $b$ ' (dotted lines).

The affine configuration is depicted by dashed lines in Fig. 11b. Next, we introduce the vector $\hat{\mathbf{u}}=\mathbf{F u}^{0}$ to denote the in-plane translation of the cell wall intersection line with respect to the affine configuration (Fig. 11b). With the displacement $\hat{\mathbf{u}}$ at hand, the four vectors defining the current microstructural configuration, $\mathbf{e}_{T}, \mathbf{n}_{1}, \mathbf{n}_{2}$, and $\mathbf{n}_{3}$, are expressed as follows:

$$
\begin{aligned}
& \mathbf{n}_{r}=\hat{\mathbf{n}}_{r}+\hat{\mathbf{u}}=\mathbf{F}\left(\mathbf{n}_{r}^{0}+\mathbf{u}^{0}\right) \quad \text { for } r=1,2,3 \\
& \mathbf{e}_{T}=\hat{\mathbf{e}}_{T}=\mathbf{F e}_{T}^{0}
\end{aligned}
$$

Thus, knowing the macroscopic deformation gradient $\mathbf{F}$ and the internal displacement $\mathbf{u}^{0}$, the current microstructural configuration is uniquely defined by Eq. (10). While $\mathbf{F}$ is typically prescribed in macrostrain-driven computations, the displacement vector $\mathbf{u}^{0}$ is a priori unknown; it will be determined from the equilibrium equations at the microscale, which are part of the local problem. In this context, it is important to note that $\mathbf{u}^{0}$ is an in-plane vector which may be written as a linear combination of any two in-plane vectors, e.g.,

$$
\mathbf{u}^{0}=u_{W} \mathbf{e}_{W}^{0}+u_{W} \mathbf{e}_{L}^{0}
$$

where $\mathbf{e}_{W}^{0}$ and $\mathbf{e}_{L}^{0}$ denote the in-plane unit vectors aligned with the $W$ - and $L$-direction, respectively. Therefore, the determination of the current microstructural configuration reduces to the computation of the displacement components $u_{W}$ and $u_{L}$.

\subsection{Strain concentration}

It follows from the three-membrane unit cell assumption that the micro-strain fields are uniform within each cell wall. The current configuration of the cell wall $r$ is defined by two vectors: $\mathbf{n}_{r}$ and $\mathbf{e}_{T}$ (Fig. 11a). In close analogy with non-linear shell element formulations 
(e.g., Belytschko et al., 1984), co-rotational, local coordinate systems are introduced to evaluate the membrane strains. For each cell wall $(r=1,2,3)$, we define three coordinate vectors $\mathbf{s}_{x}^{r}, \mathbf{s}_{y}^{r}$, and $\mathbf{s}_{z}^{r}$, where

$$
\begin{aligned}
\mathbf{s}_{x}^{r} & =\frac{\mathbf{n}_{r}}{\left|\mathbf{n}_{r}\right|} \\
\mathbf{s}_{z}^{r} & =\frac{\mathbf{n}_{r} \times \mathbf{e}_{T}}{\left|\mathbf{n}_{r} \times \mathbf{e}_{T}\right|}
\end{aligned}
$$

and

$$
\mathbf{s}_{y}^{r}=\mathbf{s}_{z}^{r} \times \mathbf{s}_{x}^{r}
$$

The unit vector $\mathbf{s}_{x}^{r}$ is always aligned with the current in-plane direction of cell wall $r$ in the deformed configuration, whereas $\mathbf{s}_{z}^{r}$ defines the unit normal to the deformed membrane. In Fig. 11a, the directions of the local coordinate vectors have been indicated for the cell wall $r=1$ (grey-shaded). Observe that the vectors $\mathbf{s}_{y}^{r}$ do not coincide with the $T$-direction when out-of-plane shear deformation is applied. Using the local coordinate vectors, we define the local deformation gradients, $\mathbf{F}^{r}$,

$$
\mathbf{F}^{r}=\left(\mathbf{n}_{r} \cdot \mathbf{s}_{x}^{r}\right) \mathbf{d}_{1} \otimes \mathbf{d}_{1}+\left(\mathbf{e}_{T} \cdot \mathbf{s}_{x}^{r}\right) \mathbf{d}_{1} \otimes \mathbf{d}_{2}+\left(\mathbf{e}_{T} \cdot \mathbf{s}_{y}^{r}\right) \mathbf{d}_{2} \otimes \mathbf{d}_{2}
$$

with $\mathbf{d}_{1}=\left[\begin{array}{ll}1 & 0\end{array}\right]^{\mathrm{T}}$ and $\mathbf{d}_{2}=\left[\begin{array}{ll}0 & 1\end{array}\right]^{\mathrm{T}}$. For each cell-wall $r$, the corresponding two-dimensional second-order tensor $\mathbf{F}^{r}$ describes the locally homogeneous deformation field of the membrane plane. Using the right Cauchy-Green deformation tensors $\left(\mathbf{F}^{r}\right)^{\mathrm{T}} \mathbf{F}^{r}$, we compute the local logarithmic strain tensors $\boldsymbol{\varepsilon}^{r}$. Formally, we write

$$
\boldsymbol{\varepsilon}^{r}=\ln \sqrt{\left(\mathbf{F}^{r}\right)^{\mathrm{T}} \mathbf{F}^{r}} \text { for } r=1,2,3
$$

where both the square root and the log-operation are applied to the eigenvalues of the tensor argument. The objectivity of the local strain tensors may be shown as follows. For a macroscopic rotation $\mathbf{Q}, \mathbf{Q} \mathbf{Q}^{\mathrm{T}}=\mathbf{1}$, we have the transformations: $\mathbf{F} \rightarrow \mathbf{Q F}, \mathbf{n}_{r} \rightarrow \mathbf{Q} \mathbf{n}_{r}$, $\mathbf{e}_{T} \rightarrow \mathbf{Q \mathbf { e } _ { T }}, \mathbf{s}_{j}^{r} \rightarrow \mathbf{Q s _ { j } ^ { r }}$, but $\mathbf{n}_{r} \cdot \mathbf{s}_{j}^{r} \rightarrow \mathbf{Q \mathbf { n } _ { r }} \cdot \mathbf{Q \mathbf { s } _ { j } ^ { r }}=\mathbf{n}_{r} \cdot \mathbf{s}_{j}^{r}$ and $\mathbf{e}_{T} \cdot \mathbf{s}_{j}^{r} \rightarrow \mathbf{Q \mathbf { e } _ { T }} \cdot \mathbf{Q \mathbf { s } _ { j } ^ { r }}=\mathbf{e}_{T} \cdot \mathbf{s}_{j}^{r}$, in what follows the frame invariance of the local deformation gradients $\mathbf{F}^{r} \rightarrow \mathbf{F}^{r}$ and of the strain tensors $\boldsymbol{\varepsilon}^{r} \rightarrow \boldsymbol{\varepsilon}^{r}$.

\subsection{Constitutive behavior at the microscale}

The material model at the microscale must be chosen in accordance with the cell wall base material. Here, we briefly recall the finite strain plane-stress constitutive equations for Levy-von Mises materials with isotropic strain-hardening. For ease of notation, the symmetric strain tensors $\boldsymbol{\varepsilon}^{r}$ are rewritten in the form of strain vectors, $\overrightarrow{\boldsymbol{\varepsilon}}^{r}, r=1,2,3$

$$
\boldsymbol{\varepsilon}^{r}=\left[\begin{array}{cc}
\varepsilon_{x}^{r} & \varepsilon_{x y}^{r} \\
\varepsilon_{x y}^{r} & \varepsilon_{y y}^{r}
\end{array}\right] \text { and } \overrightarrow{\boldsymbol{\varepsilon}}^{r}=\left[\begin{array}{lll}
\varepsilon_{x}^{r} & \varepsilon_{y}^{r} & 2 \varepsilon_{x y}^{r}
\end{array}\right]^{\mathrm{T}}
$$

Similarly, the energy-conjugate Cauchy stress tensors $\boldsymbol{\sigma}^{r}$ are represented by the vectors $\overrightarrow{\boldsymbol{\sigma}}^{r}$. Introducing the vector of local plastic strains $\overrightarrow{\boldsymbol{\varepsilon}}_{\mathrm{p}}^{r}$, the local elastic stress-strain relationship reads

$$
\overrightarrow{\boldsymbol{\sigma}}^{r}=\mathbf{C}\left(\overrightarrow{\boldsymbol{\varepsilon}}^{r}-\overrightarrow{\boldsymbol{\varepsilon}}_{\mathrm{p}}^{r}\right)
$$


$\mathbf{C}$ is the plane-stress stiffness matrix for the isotropic base material of modulus $E_{\mathrm{s}}$ and elastic Poisson's ratio $v_{\mathrm{s}}$ :

$$
\mathbf{C}=\frac{E_{\mathrm{s}}}{1-v_{\mathrm{s}}^{2}}\left\{\mathbf{e}_{1} \otimes \mathbf{e}_{1}+\mathbf{e}_{2} \otimes \mathbf{e}_{2}+v_{\mathrm{s}}\left(\mathbf{e}_{1} \otimes \mathbf{e}_{2}+\mathbf{e}_{2} \otimes \mathbf{e}_{1}\right)+\frac{1}{2}\left(1-v_{\mathrm{s}}\right) \mathbf{e}_{3} \otimes \mathbf{e}_{3}\right\}
$$

For each membrane, the local von Mises yield function $f^{r}$ reads

$$
f^{r}\left(\boldsymbol{\sigma}^{r}, s^{r}\right)=\sqrt{\left(\sigma_{x}^{r}\right)^{2}+\left(\sigma_{y}^{r}\right)^{2}-\sigma_{x}^{r} \sigma_{y}^{r}+3\left(\sigma_{x y}^{r}\right)^{2}}-s^{r}
$$

where $s^{r}$ denotes the deformation resistance at the microscale. The evolution of $s^{r}$ is governed by the non-linear hardening law

$$
\mathrm{d} s^{r}=h\left(s^{r}\right) \mathrm{d} \alpha^{r}
$$

with the hardening modulus $h=h\left(s^{r}\right)$ and the non-negative plastic multiplier $\mathrm{d} \alpha^{r}$. The direction of plastic flow at the microscale is described by the associated flow rule

$$
\mathbf{d} \boldsymbol{\varepsilon}_{p}^{r}=\frac{\partial f^{r}}{\partial \boldsymbol{\sigma}^{r}} \mathrm{~d} \alpha^{r}
$$

In addition to the evolution equations, the Kuhn-Tucker complementary conditions must be satisfied locally,

$$
\mathrm{d} \alpha^{r} \geqslant 0, \quad f^{r} \leqslant 0, \quad\left(\mathrm{~d} \alpha^{r}\right) f^{r}=0
$$

along with the consistency condition

$$
\left(\mathrm{d} \alpha^{r}\right)\left(\mathrm{d} f^{r}\right)=0 \quad \text { when } f^{r}=0
$$

In order to take plastic membrane thickness changes into account, we evaluate the plastic normal strains $\varepsilon_{z, \mathrm{p}}^{r}$ along the local $\mathbf{s}_{z}^{r}$-directions:

$$
\varepsilon_{z, \mathrm{p}}^{r}=-\left(\varepsilon_{x, \mathrm{p}}^{r}+\varepsilon_{y, \mathrm{p}}^{r}\right)
$$

\subsection{In-plane force equilibrium}

The local in-plane displacements $u_{W}$ and $u_{L}$ are chosen such that force equilibrium is satisfied in the $T-W$-plane. It follows from local force equilibrium that the sum of in-plane forces along the cell wall intersection line must be zero. Recall from the local coordinate system definition that the coordinate vectors $\mathbf{s}_{x}^{r}$ lie in the deformed $T-W$-plane and point away from the intersection line. Thus, evaluation of force equilibrium yields the following vector equation:

$$
2\left(1+\varepsilon_{z, \mathrm{p}}^{3}\right) \sigma_{x}^{3} \mathbf{s}_{x}^{3}+\sum_{r=1}^{2}\left(1+\varepsilon_{z, \mathrm{p}}^{r}\right) \sigma_{x}^{r} \mathbf{s}_{x}^{r}=\mathbf{0}
$$

The factor 2 in front of the first term accounts for the difference in initial thickness among the cell walls, i.e., the double-thickness of cell wall $r=3$. The mathematical problem defined by Eqs. (8)-(26) may be solved numerically. As a result, the in-plane displacements $u_{W}$ and $u_{L}$ are obtained along with the entire solution of the local problem, notably the local stress tensors $\boldsymbol{\sigma}^{r}$. 


\subsection{Stress homogenization}

In a final step, the macroscopic Cauchy stress tensor $\boldsymbol{\Sigma}$ is estimated based on the solution of the local problem. For this, we make use of the mean value theorem for the stress field, ${ }^{1}$

$$
\Sigma \cong \frac{1}{\Omega} \int_{\Omega} \boldsymbol{\sigma}(\mathbf{y}) \mathrm{d} \Omega
$$

where $\Omega$ denotes the current unit cell volume. The integrand $\sigma=\sigma(\mathbf{y})$ corresponds to the stress field at the microscale, expressed as a function of some local coordinate $\mathbf{y}$. For the three-membrane system, the microstructural stress field is piecewise constant and we may rewrite Eq. (27) as

$$
\boldsymbol{\Sigma}=\sum_{r=1}^{3} \boldsymbol{\sigma}_{g}^{r} \frac{\Omega^{r}}{\Omega}, \quad c^{r}=\frac{1}{\Omega} \int_{\Omega^{r}} \mathrm{~d} \Omega=\frac{\Omega^{r}}{\Omega}
$$

$c^{r}$ denotes the current volume ratio of membrane $r$, while the three-dimensional second-order tensor $\boldsymbol{\sigma}_{g}^{r}$ describes the corresponding constant local stress field in global coordinates:

$$
\boldsymbol{\sigma}_{g}^{r}=\sigma_{x}^{r} \mathbf{s}_{x}^{r} \otimes \mathbf{s}_{x}^{r}+\sigma_{y}^{r} \mathbf{s}_{y}^{r} \otimes \mathbf{s}_{y}^{r}+\sigma_{x y}^{r}\left(\mathbf{s}_{x}^{r} \otimes \mathbf{s}_{y}^{r}+\mathbf{s}_{y}^{r} \otimes \mathbf{s}_{x}^{r}\right)
$$

Neglecting elastic volume changes at the microscale, i.e., $\mathrm{d} \Omega^{r}=0$, we have

$$
c^{r}=\frac{c_{0}^{r}}{\operatorname{det} \mathbf{F}}
$$

The initial cell wall volume ratios $c_{0}^{r}$ are directly related to the relative density $\rho^{*}$ of the honeycomb material,

$$
4 c_{0}^{1}=4 c_{0}^{2}=2 c_{0}^{3}=\rho^{*}
$$

Thus, the final approximate expression for the macroscopic Cauchy stress tensor reads

$$
\boldsymbol{\Sigma} \cong \frac{\rho^{*}}{4 \operatorname{det} \mathbf{F}}\left(\boldsymbol{\sigma}_{g}^{1}+\boldsymbol{\sigma}_{g}^{2}+2 \boldsymbol{\sigma}_{g}^{3}\right)
$$

\subsection{FE-implementation}

This constitutive model for stable honeycombs has been implemented into the finite element code ABAQUS/explicit using the user-material subroutine interface for solid elements (Abaqus, 2005). For a given deformation gradient $\mathbf{F}_{n+1}$, the computational version of the constitutive model provides the macroscopic stress tensor $\boldsymbol{\Sigma}_{n+1}$ and the vector of state variables $\chi_{n+1}$ at time $t+\Delta t$, based on the state variables $\chi_{n}$ at time $t$. For the present macroscopic constitutive model, the vector of internal state variables, $\chi$, comprises 12 components: the plastic strains $\varepsilon_{x, \mathrm{p}}^{1}, \varepsilon_{y, \mathrm{p}}^{1}, \varepsilon_{z, \mathrm{p}}^{1}, \varepsilon_{x, \mathrm{p}}^{2}, \varepsilon_{y, \mathrm{p}}^{2}, \varepsilon_{z, \mathrm{p}}^{2}, \varepsilon_{x, \mathrm{p}}^{3}, \varepsilon_{y, \mathrm{p}}^{3}, \varepsilon_{z, \mathrm{p}}^{3}$, and the deformation resistances $s^{1}, s^{2}, s^{3}$. Since a strain-driven approach has been chosen for the model

\footnotetext{
${ }^{1}$ In the present formulation, the local problem is not solved exactly (the shear stresses along the intersection line are not always in static equilibrium). Thus, the local stress fields are not divergence-free for any macroscopic loading condition in what follows that Hill's macrohomogeneity equation may no longer hold. However, the mean value theorem may still be used to obtain an approximation of the macroscopic stress field.
} 
development, it is fairly straightforward to formulate the corresponding computational scheme for the stress and state variable update based on the deformation gradient at $t+\Delta t$. The overall computational procedure is schematically shown in Fig. 12. It basically reduces to finding the roots of the two-dimensional vector function $\mathbf{G}=\mathbf{G}\left(u_{W}, u_{L}\right)$,

$$
\mathbf{G}=\mathbf{G}^{1}+\mathbf{G}^{2}+2 \mathbf{G}^{3}
$$

where

$$
\begin{aligned}
& \mathbf{G}^{r}=\left[\begin{array}{ll}
G_{W}^{r} & G_{L}^{r}
\end{array}\right]^{\mathrm{T}} \\
& G_{W}^{r}=\left(1+\varepsilon_{z, \mathrm{p}}^{r}\right) \sigma_{x}^{r} \mathbf{s}_{x}^{r} \cdot \mathbf{e}_{W} \\
& G_{L}=\left(1+\varepsilon_{z, \mathrm{p}}^{r}\right) \sigma_{x}^{r} \mathbf{s}_{x}^{r} \cdot \mathbf{e}_{L}
\end{aligned}
$$

Initialize

$$
\begin{aligned}
& k=0 \\
& u_{W}^{0}=u_{W}(t) \\
& u_{L}^{0}=u_{L}(t)
\end{aligned}
$$

Iterate.

DOUNTIL $\left(\left|G_{W}\right|<T O L\right.$. AND. $\left.\left|G_{L}\right|<T O L\right)$

$$
\begin{aligned}
& \mathbf{G}=\mathbf{0}, \quad \mathbf{D G}=\mathbf{0} \\
& \text { DO } r=1,3
\end{aligned}
$$

CALL KINEMATICS $[\underbrace{\mathbf{F}_{n+1}, u_{W}^{k}, u_{L}^{k}}_{\text {in }}, \underbrace{\varepsilon_{r}^{k},\left(\mathbf{s}_{x}^{r}\right)^{k},\left(\mathbf{s}_{y}^{r}\right)^{k}}_{\text {out }}]$

CALL MEMBRANE $[\underbrace{\varepsilon_{r}^{k},\left(\mathbf{s}_{x}^{r}\right)^{k},\left(\mathbf{s}_{y}^{r}\right)^{k},\left(\boldsymbol{\varepsilon}_{p}^{r}\right)_{n}, s_{n}^{r}}_{\text {in }}, \underbrace{\mathbf{G}^{r}, \mathbf{D G}^{r}, \boldsymbol{\sigma}_{g}^{r}, \boldsymbol{\varepsilon}_{p}^{r}, s^{r}}_{\text {out }}]$

$$
\mathbf{G}=\mathbf{G}+\mathbf{G}^{r}, \quad \mathbf{D G}=\mathbf{D G}+\mathbf{D G}^{r}
$$

\section{END DO}

$$
\begin{aligned}
& u_{W}^{k+1}=u_{W}^{k}+\Delta u_{W}(\mathbf{G}, \mathbf{D G}) \\
& u_{L}^{k+1}=u_{L}^{k}+\Delta u_{L}(\mathbf{G}, \mathbf{D G}) \\
& k=k+1
\end{aligned}
$$

END DO

Update.

$$
\begin{aligned}
& \left(\boldsymbol{\varepsilon}_{p}^{r}\right)_{n+1}=\boldsymbol{\varepsilon}_{p}^{r}, \quad s_{n+1}^{r}=s^{r} \\
& \Sigma_{n+1}=\frac{\rho^{*}}{4 \operatorname{det} \mathbf{F}_{n+1}}\left(\sigma_{g}^{1}+\sigma_{g}^{2}+2 \sigma_{g}^{3}\right)
\end{aligned}
$$

Fig. 12. Computational procedure. In addition to the variables described in the model formulation, the computational model makes use of the numerically-determined Jacobian $\mathbf{D G}=\mathbf{d G} / \mathbf{d u}(2 \times 2$ matrix $)$ of the vector function $\mathbf{G}(\mathbf{u})$. 
This solution $\left(u_{W}, u_{L}\right)$ is obtained using a Broyden algorithm (e.g., Press et al., 1992). The subroutine MEMBRANE (Fig. 12) performs the stress update at the microscale using a standard plane-stress $J_{2}$-plasticity algorithm (e.g., Simo and Hughes, 1998).

\section{Model calibration and verification}

In this section, all previously performed virtual experiments on sandwich sheets are simulated using the new continuum model for the honeycomb core material. Using a practical engineering approach, the material model is calibrated based on a single virtual experiment and then validated by the remaining experimental results.

\subsection{Calibration}

According to the model formulation, the honeycomb material model behavior is determined by the relative density $\rho^{*}$ and by the function $h(s)$ which characterizes the isotropic hardening behavior of the cell wall material. Furthermore, the initial orientation of the orthotropy axes $L$ and $W$ must be specified. The relative density of a honeycomb material can be easily measured and is typically known, while the hardening function $h(s)$ is usually a priori unknown. From a theoretical point of view, the function $h(s)$ corresponds to the first derivative of the uniaxial stress-strain curve for the cell wall material, $h(s)=\mathrm{d} s / \mathrm{d} \alpha$, and thus, it may be determined from uniaxial tensile tests on the cell wall material. However, from an engineering perspective, it is recommended to determine $h(s)$ indirectly from an out-of-plane shear test on the honeycomb sandwich which guarantees optimal model predictions for this specific loading case. In the present virtual experiments, the honeycomb has a relative density of $\rho^{*}=12.5 \%$. The out-of-plane shear test along the $W$-direction on the initially undeformed sandwich material is chosen to calibrate the hardening function $h(s)$. For this specific loading condition, close agreement of the continuum model with the detailed finite element model (virtual experiments) is obtained when using the slope of the dashed stress-strain curve labeled $s(\alpha)$ in Fig. 2. Note that this curve lies above the 'real' stress-strain curve of the cell wall material (Hastelloy X) which indicates that the continuum model would underestimate the core strength if the "real" stress-strain curve was used. Recall that the continuum model has been developed such that it provides a suitable mathematical structure to describe first-order effects. For instance, the above used model of the sandwich structure satisfies the compatibility between the core and face sheets at the macroscopic level, but not locally. Satisfying local compatibility (i.e., displacement continuity between the honeycomb cell walls and the face sheets) requires additional plastic work which is not captured by the continuum model. In most practical applications, it is fairly difficult to obtain the 'real' stress-strain curve directly from experiments on the cell wall material. Instead, the dashed curve labeled $s(\alpha)$ will be obtained indirectly through macroscopic experiments such as the out-of-plane shear test described above.

\subsection{Verification}

It is emphasized that only the $W$-shear test on the undeformed honeycomb material $\left(E_{L L}=0\right)$ has been used for calibration. In this subsection, the predictive capabilities of the continuum model for in-plane loading along the $W$ - and $L$-direction as well as for shear along the $L$-direction (including history effects) are evaluated. 
As far as in-plane loading is concerned, excellent agreement is observed of the model predictions and the detailed FEA results (Fig. 6). For both in-plane loading along the $W$ - and $L$-direction, the continuum model estimates (open circles) follow closely the solid curves of the detailed FE simulations. The good accord for compressive loading indicates that the continuum model captures the low-resistance deformation modes of the core material. At the same time, the model provides reasonable predictions of significant internal energy variations within the honeycomb core material for in-plane tensile loading. Observe that the response curves of the detailed and continuum model nearly coincide for tensile loading (Fig. 6).

The continuum model which has been calibrated for shear loading along the $W$-direction also provides a good estimate for the material response to shear loading along the $L$-direction (Fig. 10). The accurate agreement for both cases demonstrates the model's capability to represent the pronounced orthotropy of hexagonal honeycombs. Fig. 10 also shows the model predictions of the out-of-plane shear response after in-plane loading. In the case of a tensile loading history along the $L$-direction $\left(E_{L L}=0.4\right)$, the model prediction of the out-of-plane shear stress along the $W$-direction is still very accurate, while it overpredicts the increase in shear resistance due to compressive in-plane loading $\left(E_{L L}=-0.4\right)$. Similar observations are made regarding the effect of the $W$-in-plane loading history on the out-of-plane shear resistance in the $L$-direction (Fig. 10a). In this loading case, the maximum differences between the results of the continuum and detailed model are even considerably smaller.

\section{Yield surface evolution}

The constitutive model accounts for the macroscopic hardening/softening due to both true strain-hardening of the base material and geometrical changes at the microscale. In this section, we discuss the evolution of the anisotropic macroscopic yield surface to illustrate this important model property. Our attention is limited to the subspace of pure outof-plane shear loading, where $\Sigma_{W W}=\Sigma_{L L}=\Sigma_{T T}=\Sigma_{W L}=0$. In this subspace (the $\Sigma_{W T}-\Sigma_{L T}$-plane), the otherwise six-dimensional yield surface of the honeycomb core material may be represented by a closed two-dimensional envelope.

The evolution of the initial shear yield surface ( $\Sigma_{W T}-\Sigma_{L T}$-plane) is investigated for the aforementioned in-plane loading histories: uniaxial compression and tension along the $L$ - and $W$-direction, respectively. For these specific loading conditions, the microstructure remains orthotropic and the change of cell wall orientation may be described by the evolution of some selected components of the normalized cell wall orientation vectors $\mathbf{s}_{x}^{1}$ and $\mathbf{s}_{x}^{2}$, respectively. When the three-orthotropy axes are aligned with the global coordinate directions, i.e., $\mathbf{e}_{W}=\mathbf{e}_{1}, \mathbf{e}_{L}=\mathbf{e}_{2}$, and $\mathbf{e}_{T}=\mathbf{e}_{3}$, we have $\mathbf{s}_{x}^{3}(t)=\mathbf{e}_{2}$ and $\mathbf{s}_{y}^{r}=\mathbf{e}_{3}, r=1,2,3$. Furthermore, the cell wall orientation vectors may be written as

$$
\begin{aligned}
& \mathbf{s}_{x}^{1}=\left[\begin{array}{lll}
-a_{1} & -a_{2} & 0
\end{array}\right] \\
& \mathbf{s}_{x}^{2}=\left[\begin{array}{lll}
a_{1} & -a_{2} & 0
\end{array}\right]
\end{aligned}
$$

where the two non-negative scalar functions $a_{1}(t)>0$ and $a_{2}(t)>0$ are introduced to describe the evolution of the normalized in-plane orientation vectors.

In order to obtain macroscopic yield conditions for out-of-plane shear, the local stress tensors $\boldsymbol{\sigma}_{g}^{r}$ are expressed as a function of the macroscopic shear stresses $\Sigma_{W T}$ and $\Sigma_{L T}$. For 
infinitesimal deformations (initial yield of the orthotropic microstructure), out-of-plane shear loads generate pure shear at the microscale, i.e.

$$
\boldsymbol{\sigma}_{g}^{r}=\sigma_{x y}^{r}\left(\mathbf{s}_{x}^{r} \otimes \mathbf{s}_{y}^{r}+\mathbf{s}_{y}^{r} \otimes \mathbf{s}_{x}^{r}\right) \quad \text { while } \sigma_{x x}^{r}=\sigma_{y y}^{r}=0
$$

Now, consider shear loading along the positive $W$-direction, $\Sigma_{W T} \geqslant 0$. We have $\sigma_{x y}^{3}=0$ and $\tau_{\mathrm{s}}^{(w)}:=-\sigma_{x y}^{1}=\sigma_{x y}^{2}>0$, where $\tau_{\mathrm{s}}^{(W)}$ denotes the shear stress in a single-thickness cell wall due to macroscopic $W$-shear loading. Upon evaluation of Eq. (39), we find

$$
\boldsymbol{\sigma}_{g}^{1}+\boldsymbol{\sigma}_{g}^{2}=2 a_{1} \tau_{\mathrm{s}}^{(w)}\left(\mathbf{e}_{1} \otimes \mathbf{e}_{3}+\mathbf{e}_{3} \otimes \mathbf{e}_{1}\right)
$$

Using this expression in Eq. (32) yields

$$
\tau_{\mathrm{s}}^{(W)}=\frac{2 \operatorname{det} \mathbf{F}}{\rho^{*} a_{1}} \Sigma_{W T} .
$$

Next, consider shear loading along the $L$-direction, $\Sigma_{L T} \geqslant 0$. This type of loading generates local shear stresses in the single-thickness walls, $\tau_{\mathrm{s}}^{(L)}:=-\sigma_{x y}^{1}=-\sigma_{x y}^{2}>0$, and in the double-thickness wall, $\tau_{\mathrm{d}}^{(L)}:=\sigma_{x y}^{3}>0$. It follows from evaluating Eqs. (39) and (32) that:

$$
\Sigma_{L T}=\frac{\rho^{*}}{2 \operatorname{det} \mathbf{F}}\left(a_{2} \tau_{s}^{(L)}+\tau_{\mathrm{d}}^{(L)}\right) .
$$

For shear loading in $L$-direction, the continuum model (which does not generally satisfy equilibrium for the shear stresses along the intersection line) suggests the following relationship between the shear stresses at the microscale:

$$
\tau_{\mathrm{s}}^{(L)}=a_{2} \tau_{\mathrm{d}}^{(L)}
$$

Combining Eqs. (41) and (42) yields

$$
\begin{aligned}
\tau_{\mathrm{s}}^{(L)} & =\frac{2 \operatorname{det} \mathbf{F}}{\rho^{*}} \frac{a_{2}}{a_{2}^{2}+1} \Sigma_{L T} \\
\tau_{\mathrm{d}}^{(L)} & =\frac{2 \operatorname{det} \mathbf{F}}{\rho^{*}} \frac{1}{a_{2}^{2}+1} \Sigma_{L T}
\end{aligned}
$$

For combined out-of-plane shear loading, the maximum local shear stresses at the microscale are given by superposition. In other words, the highest shear stress in a single-thickness wall (either $r=1$ or $r=2$ ) is

$$
\max \left|\tau_{\mathrm{s}}\right|=\tau_{\mathrm{s}}^{(w)}+\tau_{\mathrm{s}}^{(L)}=\frac{2 \sqrt{3} \operatorname{det} \mathbf{F}}{\rho^{*}}\left(\frac{\left|\Sigma_{W T}\right|}{a_{1}}+\frac{a_{2}\left|\Sigma_{L T}\right|}{a_{2}^{2}+1}\right)
$$

while for the double-thickness wall $(r=3)$, we have

$$
\max \left|\tau_{\mathrm{d}}\right|=\frac{2 \sqrt{3} \operatorname{det} \mathbf{F}}{\rho^{*}} \frac{\left|\Sigma_{L T}\right|}{a_{2}^{2}+1}
$$

At the microscale, the elastic domain is enclosed by the von Mises yield surfaces of the cell wall material. For pure shear loading, the local yield conditions are $(r=1,2,3)$

$$
f^{r}=\sqrt{3}\left|\sigma_{x y}^{r}\right|-s^{r}=0
$$

Recall that our attention is limited to the effect of uniaxial in-plane loading histories on the macroscopic yield surface, where we have $s^{1}(t)=s^{2}(t)$. Thus, using expressions (46) and 
(47) for the shear stresses in Eq. (48), we obtain two conditions in terms of $\Sigma_{W T}$ and $\Sigma_{L T}$ which determine the onset of yield of the honeycomb material. Formally, we write

$$
\begin{aligned}
& F^{\mathrm{I}}\left(\Sigma_{W T}, \Sigma_{L T}\right):=\frac{\left|\Sigma_{W T}\right|}{K_{W T}^{\mathrm{I}}}+\frac{\left|\Sigma_{L T}\right|}{K_{L T}^{\mathrm{I}}}-1 \leqslant 0 \\
& F^{\mathrm{II}}\left(\Sigma_{L T}\right):=\frac{\left|\Sigma_{L T}\right|}{K_{L T}^{\mathrm{II}}}-1 \leqslant 0
\end{aligned}
$$

where $F^{\mathrm{I}}$ and $F^{\mathrm{II}}$ may be considered as macroscopic yield functions which are determined by the macroscopic deformation resistances $K_{W T}^{\mathrm{I}}, K_{L T}^{\mathrm{I}}$, and $K_{L T}^{\mathrm{II}}$ :

$$
K_{W T}^{\mathrm{I}}=\frac{a_{1} \rho^{*} s^{1}}{2 \sqrt{3} \operatorname{det} \mathbf{F}}, \quad K_{L T}^{\mathrm{I}}=\frac{\left(1+a_{2}^{2}\right) \rho^{*} s^{1}}{2 \sqrt{3} a_{2} \operatorname{det} \mathbf{F}}, \quad K_{L T}^{\mathrm{II}}=\frac{\left(1+a_{2}^{2}\right) \rho^{*} s^{3}}{2 \sqrt{3} \operatorname{det} \mathbf{F}}
$$

It is emphasized that the macroscopic yield functions $F^{\mathrm{I}}$ and $F^{\mathrm{II}}$ account for microstructural evolution due to

(i) true strain-hardening of the base material: $s^{1}(t)$ and $s^{3}(t)$;

(ii) geometrical changes: $a_{1}(t), a_{2}(t), \operatorname{det} \mathbf{F}(t)$.

In the first quadrant of the $\Sigma_{W T}-\Sigma_{L T}$-plane, the macroscopic yield envelope corresponds to two intersecting straight lines (Fig. 13). The yield condition $F^{\mathrm{I}}=0$ associated with the yielding of the thin cell walls is a straight line intersecting both the $\Sigma_{W T}$ and $\Sigma_{L T}$ axes. The condition $F^{\mathrm{II}}=0$ imposes a restriction on the shear stress $\Sigma_{L T}$ only, since shear along the $W$-direction does not cause any stresses in the double-thickness cell walls.

The history of the model parameters $a_{1}, a_{2}, \operatorname{det} \mathbf{F}, s^{1}$ and $s^{3}$ has been extracted from the continuum model simulations. Subsequently, the macroscopic deformation resistances have been determined for uniaxial in-plane loading along the $W$-direction (at $E_{W W}=-0.4$ and $E_{W W}=0.4$ ) and along the $L$-direction ${ }^{2}$ (at $E_{L L}=-0.25$ and $\left.E_{L L}=0.4\right)$. The corresponding yield envelopes are depicted in Fig. 14. Irrespective of the loading direction, in-plane loading causes an elongation of the shear yield locus along either coordinate axis, which may be considered as an evolution of the material orthotropy. As mentioned before, the orientation of the cell walls has a significant effect on the out-of-plane shears strengths. From a geometrical point of view, an increase of the cell wall orientation angle $\theta$ (Fig. 1), increases the macroscopic shear strength along the $L$-direction while the shear resistance in the $W$-direction decreases. Thus, since both tension along the $L$-direction and compression in the $W$-direction lead to an increase of $\theta$ (Fig. $7 \mathrm{~b}$ and c), we observe an elongation of the shear yield surface along the $\Sigma_{L T}$-axis for $E_{L L}=0.4$ or $E_{W W}=-0.4$ (Fig. 14). Conversely, a pronounced elongation along the $\Sigma_{W T}$-axis is observed when the sandwich material is preloaded in the opposite directions, i.e., $E_{L L}=-0.25$ or $E_{W W}=0.4$. In addition to geometrical effects, the yield surface changes due to material hardening at the microscale. Recall that the unconstrained deformation modes of the core material and face sheets are more compatible for compressive than for tensile in-plane loading (Fig. 5), which results in more pronounced base material

\footnotetext{
${ }^{2}$ Note that $a_{2}$ becomes negative for $E_{L L}<-0.25$. The macroscopic shear strength may also be calculated for negative $a_{2}$. However, we limit our attention to the limiting case of $E_{L L}=-0.25$ in order to omit additional derivations of the same type as those shown above.
} 


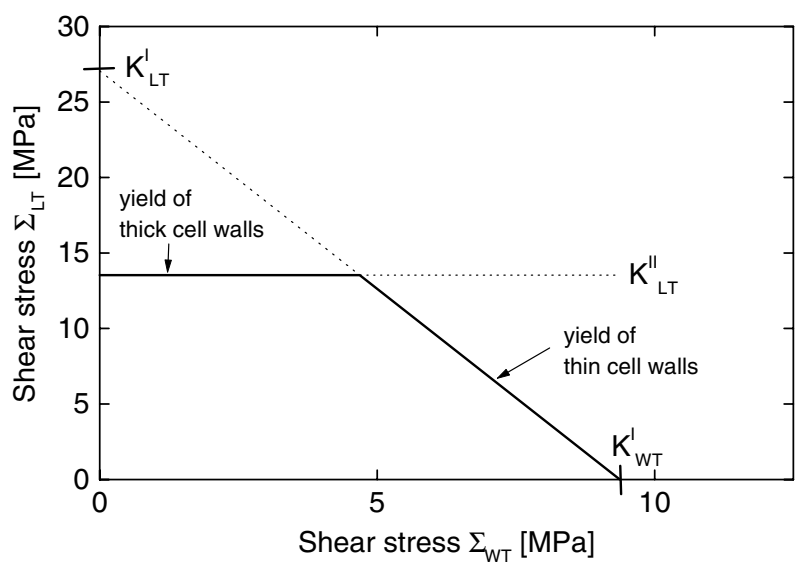

Fig. 13. Macroscopic yield surface for pure out-of-plane shear (initially undeformed microstructure).

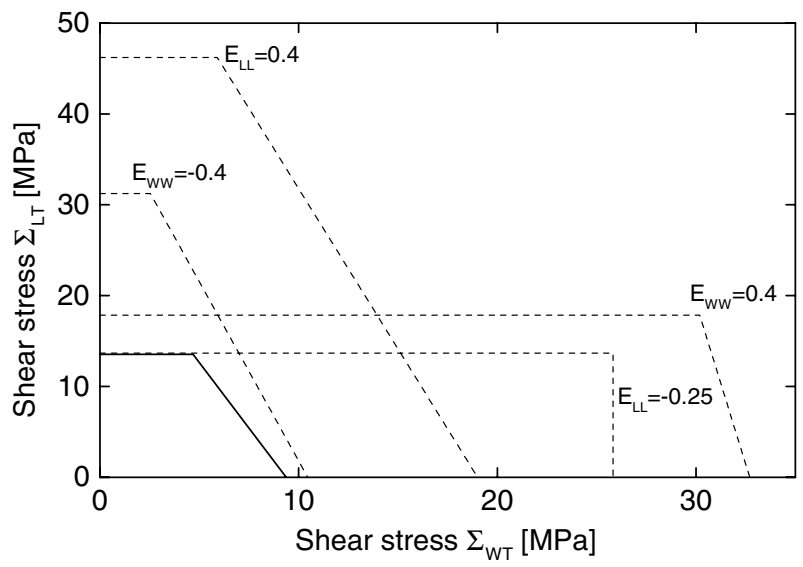

Fig. 14. Macroscopic yield surface for pure out-of-plane shear and its evolution due to uniaxial in-plane loading along the $W$ - and $L$-direction, respectively.

hardening in the latter case. Therefore, for similar orthotropy evolution directions, the resultant yield surfaces for uniaxial compression loading histories $\left(E_{W W}=-0.4\right.$ and $\left.E_{L L}=-0.25\right)$ lie inside those for uniaxial tension $\left(E_{L L}=0.4\right.$ and $\left.E_{W W}=0.4\right)$. Another interesting feature of the yield surface evolution is observed for in-plane compression along the $L$-direction. At $E_{L L}=-0.25$, we have $a_{2} \cong 0$ which means that the initially hexagonal cell structure has transformed into a square honeycomb. As a result, the line $F^{\mathrm{I}}=0$ becomes perpendicular to $F^{\mathrm{II}}=0$ because shear along the $L$-direction no longer generates stresses in the thin-walls. A more detailed discussion of the interaction between microstructural changes and the macroscopic yield surface evolution is beyond the scope of this paper. However, it is concluded that the macroscopic out-of-plane shear yield surface changes dramatically due to in-plane loading. This evolution involves both changes in size and shape of the yield surface. 


\section{Conclusions}

The large deformation behavior of a sandwich material with a thick-walled metallic honeycomb core has been studied by means of detailed finite element analysis. Motivated by the simulation results, a simple unit cell comprised of three intersecting membranes has been proposed and used to develop a macroscopic constitutive model of the honeycomb core material. The material model has been validated for large in-plane loading and out-of-plane shear loading. Unlike phenomenological constitutive models, the present micro-mechanics-based model exhibits some predictive capabilities. Due to the universal applicability of the underling microstructural model, it is expected to yield reasonable results for arbitrary loading histories.

The macroscopic plasticity of metallic honeycombs appears to be fundamentally different from solid materials, which requires the development of a suitable mathematical framework. Here, a micromechanical approach has been chosen which includes conventional constitutive equations at the microscale along with strain localization and stress homogenization relationships. This two-scale nature of the model is maintained, in particular due to the ease of its computational implementation. The macroscopic yield surface has been derived for pure shear loading. The results have demonstrated that microstructural changes due to in-plane loading have a considerable influence on the macroscopic yield locus, both its shape and size.

\section{Acknowledgements}

Dr. Michele Brun from Ecole Polytechnique is thanked for his help on the numerical implementation of the constitutive model. The financial support by the Cambridge-MIT Institute is gratefully acknowledged.

\section{References}

Abaqus, 2005. Reference manuals of Abaqus 6.4. Abaqus Inc., Providence, RI.

Belytschko, T., Lin, J., Tsay, C.S., 1984. Explicit algorithms for nonlinear dynamics of shells. Computer Methods in Applied Mechanics and Engineering 42, 225.

Doyoyo, M., Wierzbicki, T., 2003. Experimental studies on the yield behavior of ductile and brittle aluminum foams. International Journal of Plasticity 19 (8), 1195.

Gibson, L.J., Ashby, M.F., 1997. Cellular Solids: Structure and Properties, second ed. Cambridge University Press, Cambridge.

Gurtin, M.E., 1981. An Introduction to Continuum Mechanics. Academic Press, New York.

Hong, S.-T., Pan, J., Tyan, T., Prasad, P., 2006. Quasi-static crush behavior of aluminum honeycomb specimens under compression dominant combined loads. International Journal of Plasticity 22, 73-109.

Hong, S.-T., Pan, J., Tyan, T., Prasad, P., 2006. Quasi-static crush behavior of aluminum honeycomb specimens under non-proportional compression-dominant combined loads. International Journal of Plasticity 22 (6), 1062-1086.

Mohr, D., Doyoyo, M., 2004. Large plastic deformation of metallic honeycomb: orthotropic rate-independent constitutive model. International Journal of Solids and Structures 41 (16-17), 4435.

Mohr, D., Straza, J., 2005. On the development of formable all-metal sandwich sheets for automotive applications. Advanced Engineering Materials 7 (4), 243.

Okumura, D., Ohno, N., Nogushi, H., 2004. Elastoplastic microscopic bifurcation and post-bifurcation behavior of periodic cellular solids. Journal of the Mechanics and Physics of Solids 52, 641.

Papka, S.D., Kyriakides, S., 1999. Biaxial crushing of honeycombs, Part I: Experiments. International Journal of Solids and Structures 36 (29), 4367. 
Press, W.H., Flannery, B.P., Teukolsky, S.A., Vetterling, W.T., 1992. Numerical Recipes in Fortran. Cambridge University Press, Cambridge, UK.

Simo, J.C., Hughes, T.J.R., 1998. Computational Inelasticity. Springer, New York.

Triantafyllidis, N., Schraad, M.W., 1998. Onset of failure in aluminum honeycombs under general in-plane loading. Journal of the Mechanics and Physics of Solids 46 (6), 1089.

Wang, A.-J., McDowell, D.L., 2005. Yield surfaces of various periodic metal honeycombs at intermediate relative density. International Journal of Plasticity 21, 285.

Wang, D.-A., Pan, J., 2006. A non-quadratic yield function for polymeric foams. International Journal of Plasticity 22 (3), 434-458.

Xue, Z., Vaziri, Hutchinson, J.W., 2005. Non-uniform hardening constitutive model for compressible orthotropic materials with application to sandwich plate cores. Computer Modeling in Engineering \& Sciences 10 (1), 79 95.

Zhang, J., Ashby, M.F., 1992. Buckling of honeycombs under in-plane biaxial stresses. International Journal of Mechanical Sciences 34 (6), 491.

Zhang, T.G., Lee, J., 2003. A plasticity model for cellular materials with open-celled structure. International Journal of Plasticity 19 (6), 749.

Zhao, H., Gary, G., 1998. Crushing behavior of aluminum honeycombs under impact loading. International Journal of Impact Engineering 21 (10), 827. 